\title{
Measurement of flow parameters in a Taylor- Couette configuration using UDV measurement technology
}

Heiko MEIRONKE, Daniel KLEMBT

DOI: 10.30464/jmee.2019.3.3.259

Cite this article as:

Meironke H., Klembt D. Measurement of flow parameters in a Taylor-Couette configuration using UDV measurement technology. Journal of Mechanical and Energy Engineering, Vol. 3(43), No. 3, 2019, pp. 259-266.

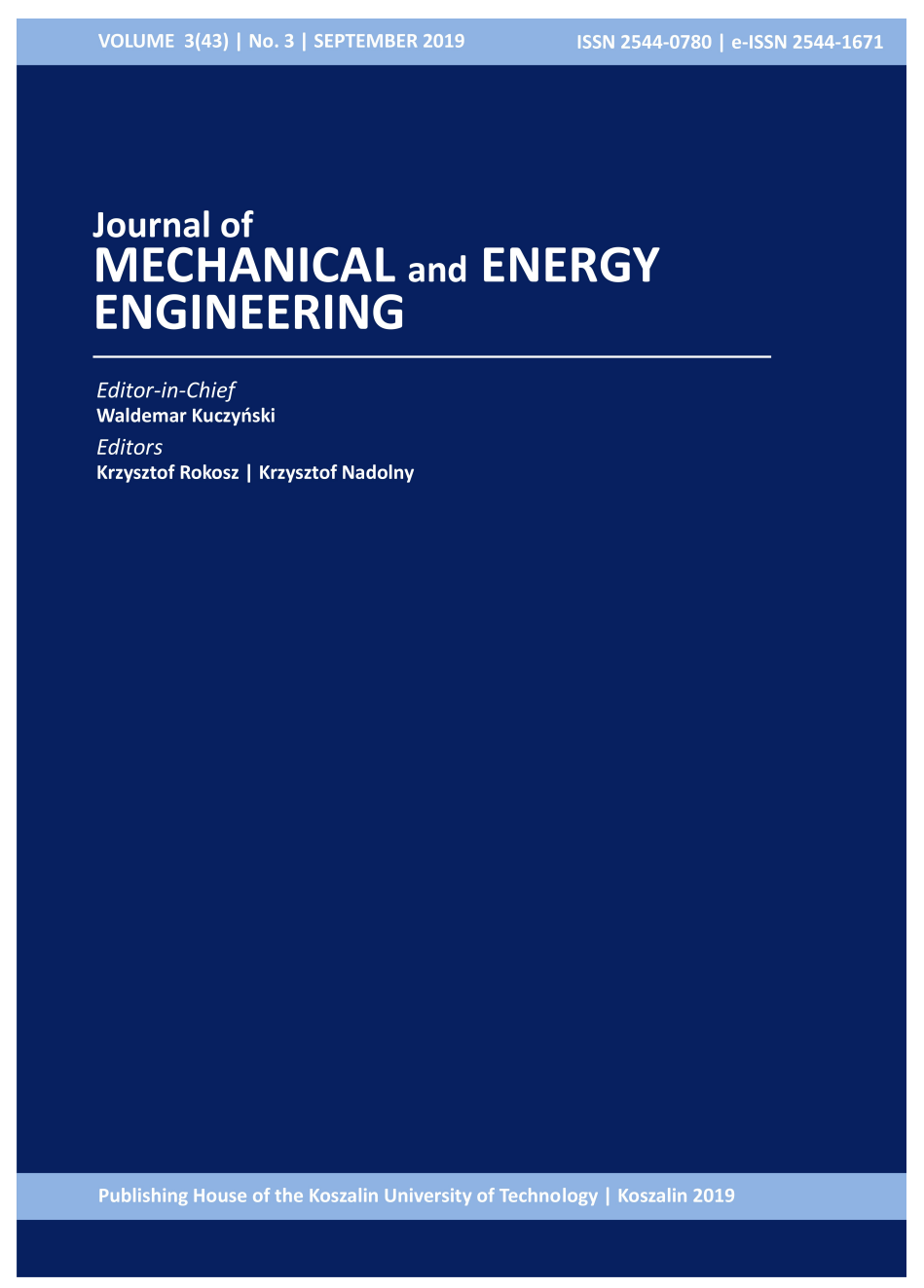

\section{Journal of Mechanical and Energy} Engineering

Website: jmee.tu.koszalin.pl

ISSN (Print): 2544-0780

ISSN (Online): 2544-1671

Volume: 3(43)

Number: 3

Year: 2019

Pages: 259-266

\section{Article Info:}

Received 12 November 2019

Accepted 10 December 2019

\section{Open Access}

This article is distributed under the terms of the Creative Commons Attribution 4.0 (CC BY 4.0) International License (http://creativecommons.org/licenses/by/4.0/), which permits unrestricted use, distribution, and reproduction in any medium, provided you give appropriate credit to the original author(s) and the source, provide a link to the Creative Commons license, and indicate if changes were made. 


\title{
MEASUREMENT OF FLOW PARAMETERS IN A TAYLOR-COUETTE CONFIGURATION USING UDV MEASUREMENT TECHNOLOGY
}

\author{
Heiko MEIRONKE $^{1 *}$, Daniel KLEMBT ${ }^{2}$ \\ 1* Faculty of Mechanical Engineering, Department of Fluid Mechanics and Apparatus Engineering, \\ University of Applied Science Stralsund, Zur Schwedenschanze 15, 18435, Stralsund, Germany, \\ e-mail: heiko.meironke@hochschule-stralsund.de \\ ${ }^{2}$ Faculty of Mechanical Engineering, Department of Fluid Mechanics and Apparatus Engineering, \\ University of Applied Science Stralsund, Zur Schwedenschanze 15, 18435, Stralsund, Germany, \\ e-mail: daniel.klembt@hochschule-stralsund.de
}

(Received 6 December 2019, Accepted 29 October 2019)

\begin{abstract}
In the context of the investigations of multiphase flows, e.g. in cooperation with the local brewery, the convective transport phenomena during the fermentation are investigated. Due to the strong turbidity of the medium, the measurement of velocity profiles is complicated. The difficulties of an investigation with a biological fermentation fluid are the many complex interactions between the different three phases (solid, gas, liquid). Furthermore, natural convection processes are superimposed by rising gas bubbles and the high turbidity of the fluid only allow an acoustic velocity measurement. This leads to high requirements for the measurement technology and the following evaluation. In previous investigation, ultrasonic transducers are used for the non-contact determination of velocity fields in fluids. The results of these past projects show that the measurement signals of the ultrasonic transducers used can be influenced by many factors. In order to verify the results of the transducers and to investigate the existing uncertainties, a flow configuration with a relatively stable reproducible flow pattern is required. In this study, a calibration system for ultrasonic transducers is developed, manufactured and validated by means of optical measurement technology such as the LDA. The experimental setup in this study produces a constantly reproducible Taylor Couette fluid flow. Geoffrey I. Taylor observed in 1923 that at a certain Reynolds number regular ring vortices are superimposed on the base flow. Along the axis, these vortices occur at the same distance, but with an alternating sense of rotation. Finally, a measurement using Ultrasonic Doppler Velocimetry in a model fluid will be compared with an optical measurement technique.
\end{abstract}

Keywords: ultrasonic doppler velocimetry, laser doppler anemometry, Taylor-Couette flow

\section{INTRODUCTION}

In the context of the investigations of multiphase flows in biological media, which are carried out in different projects, e.g. in cooperation with the local brewery, the convective transport phenomena during fermentation are analysed [1-4].

Due to the strong turbidity of the multiphase fluid, the measurement of velocity profiles is complicated. Optical measurement methods such as Particle Image Velocimetry or Laser Doppler Anemometry (LDA) cannot be used due to the turbidity. For this reason, the measurement is carried out with the use of acoustic measurement technology, by means of the Ultrasound Doppler Velocimetry (UDV). In this method, ultrasonic transducers are used for the non-contact measurements of velocity fields in fluids. The results of past projects show that the measurement signals of the ultrasonic transducers used can be influenced by many factors. In order to verify the results of the transducers and to investigate the existing uncertainties, a flow configuration with a relatively stable reproducible flow pattern is required. 
In this paper, a test setup for ultrasonic transducers is developed, manufactured and validated by means of optical measurement technology such as the LDA. The experimental setup generates a constant and reproducible fluid flow, which can be used to evaluate the signal behaviour of the velocity profile measured with UDV in a very detailed manner.

A possible flow configuration that fulfils these conditions is the Taylor-Couette flow. The advantage of this flow is the relatively simple experimental setup. The system consists of an inner and an outer cylinder in which one of the two cylinders rotates at a specified speed. In practice, such systems are used as rotary viscometers or in chemical process engineering as Taylor-Couette reactor.

Taylor vortexes are formed when the speed of the inner cylinder is increased. The consequence of the faster rotation is that the fluid in the vicinity of the inner cylinder get a greater centrifugal force than the slower flow in the outer part of the gap. Geoffrey I. Taylor observed in 1923 that from a certain Reynolds number, torus-shaped vortices are superimposed on the basic flow [5-12]. Along the rotational axis, these vortices occur at the same distance, but with an alternating sense of rotation.

In the first studies with this experimental setup, a transparent model fluid is used for a better evaluation of the signal behaviour. In addition to the acoustic profile measurements, simultaneous measurements using LDA are carried out and finally the measured velocity profiles are compared.

\section{EXPERIMENTAL SETUP}

\subsection{Experimental Arrangement}

The main component of the test setup is the Taylor-Couette system shown in Fig. 1 and described below in more detail.

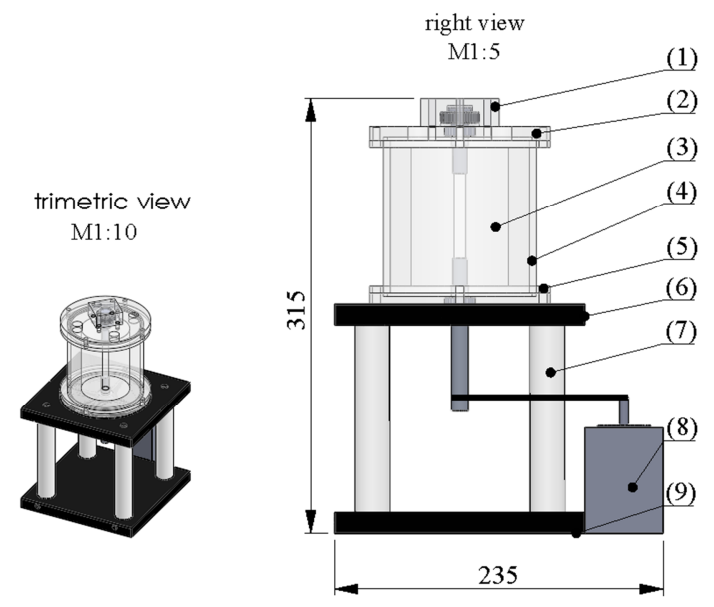

Fig. 1. Setup of the Taylor-Couette system
The external (4) and internal (3) cylinders are the main structural parts of the Taylor-Couette system, as they are essential for generating the Taylor-Couette flow. The inner diameter of the outer cylinder is 100 $\mathrm{mm}$ and the outer diameter of the inner cylinder is 70 $\mathrm{mm}$. The width of the gap is therefore $15 \mathrm{~mm}$. The wall thickness of the outer cylinder is $5 \mathrm{~mm}$. The inner cylinder is $10 \mathrm{~mm}$ smaller than the outer cylinder in order to maintain a certain distance from the ground and the cover so that rotation is possible. In addition, the inner cylinder has a thread cut for the shaft on the top and bottom.

The retaining rings (5) are glued to the head and foot of the outer cylinder so that the outer cylinder can be bolted to the base and cover with four bolts and counternuts. The two retaining rings are identical. In the base plate (6) is a hole for the drive shaft and a radial shaft seal. This base plate is connected via four columns (7) to another plate (9), which is finally attached to the test table. The cover (2) also offers mounting options for the ultrasonic transducers. Four threaded holes are provided on the underside of the plate for this purpose. The adjacent cavities are arranged so that they cover the entire gap between the inner and outer cylinders. In order to achieve the desired Taylor-Couette flow, a motor with appropriate control is required. The requirements on the motor are a high resolution and corresponding controllability in the low speed range. A stepper motor with a holding torque of $1.27 \mathrm{Nm}$ and a resolution of $0.9^{\circ}$ at $20-200$ $\mathrm{rpm}$ is used. The speed of the motor can be continuously adjusted at the corresponding potentiometer.

The Ultrasonic Doppler measuring technique measures the velocity according to the scheme shown in Fig. 2. The velocity $v$ of a flow in the direction of the transducer is represented by a negative signal, while a flow in the direction of the propagating ultrasonic wave is represented by a positive signal.

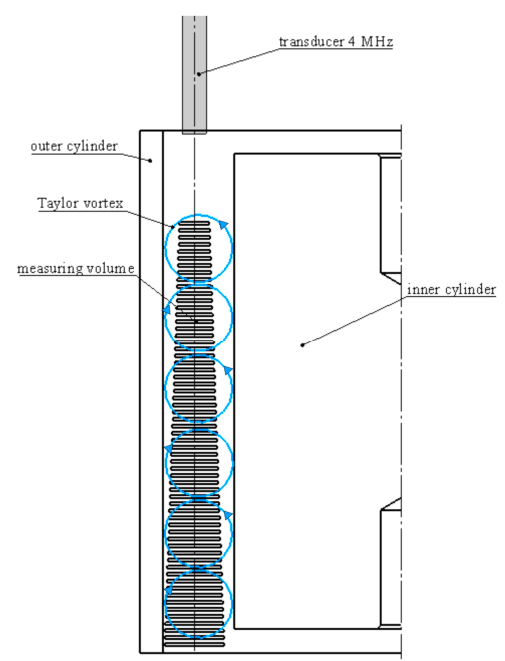

Fig. 2. Schematic representation of the measuring window of the Ultrasonic Doppler measuring technique 


\subsection{Flow measurement Ultrasonic Doppler Velocimetry}

The Ultrasonic Doppler measuring technique is an acoustic flow measuring system which can noninvasively measure flow fields. With this measuring method it is possible to measure transient velocity profiles in a flow along the beam axis.

This is possible by the measurement and subsequent evaluation of the Doppler frequencies as a function of time. Immediately after the transmission of an ultrasonic pulse (with defined basic frequency), the transducer switches to reception and waits for the reflected, frequency-shifted response signal, which reaches the probe after a defined time (see equation (1)):

$$
t=\frac{2 \cdot x}{a}[\mathrm{~s}] .
$$

When evaluating the response signal, the running time of the pulse and the spatially resolved Doppler frequency are then taken into account. Velocity information of particles which are further away on the beam axis are recorded later by the transducer and consequently assigned to a more distant measuring volume on the beam axis. The average flow velocity can then be calculated from the respective Doppler frequencies and the difference between the transmit and receive frequencies of the ultrasonic signal (see equation (2)):

$$
v_{P}=\frac{a \cdot\left(f_{T}-f_{0}\right)}{2 \cdot f_{0}}\left[\frac{\mathrm{m}}{\mathrm{s}}\right] .
$$

During evaluation, a weighted average velocity can then be assigned to each measurement volume. A simplified representation of the beam characteristic, the measurement volume dimensions and the measurement window dimensions are shown in Fig. 3.

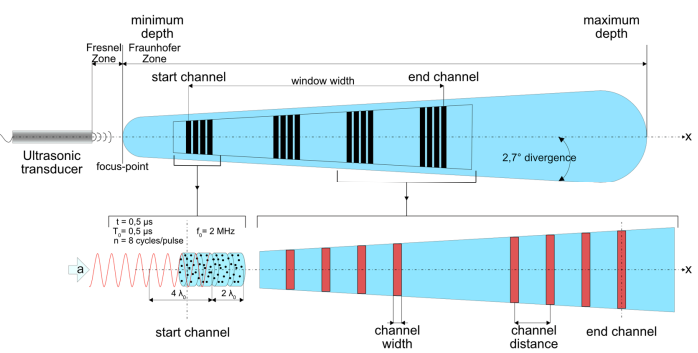

Fig. 3. Schematic representation of the beam characteristic, the measurement volume and the measurement window dimensions of a transducer

The area immediately after the transducer is called the Fresnel zone, in which no measurements are possible. In the subsequent Fraunhofer zone, the measuring field widens at a defined angle. Transducers with a base frequency of $4 \mathrm{MHz}$ were used for the measurements in this study. Due to their radiation characteristics, these transducers have a Fresnel zone of $16.9 \mathrm{~mm}$, an ultrasonic exit diameter (active diameter) of $5 \mathrm{~mm}$ and a resulting beam divergence of $2.2^{\circ}$ along the beam axis. At a constant sound velocity and base frequency of the transducers, the product of the maximum penetration depth and the maximum measurable velocity is also constant.

\subsection{Taylor-Couette flow}

According to Maurice M. A. Couette, who used this experimental unit of similar design for viscosity measurement, the basic form of the resulting flow is called circular Couette flow. When the inner cylinder rotates slowly, the flow can be described as a flat laminar flow - the Couette flow. The Couette flow (simple shear flow) is a stationary laminar flow of a liquid between two parallel flat walls that move relative to each other in their plane (see Fig. 4).

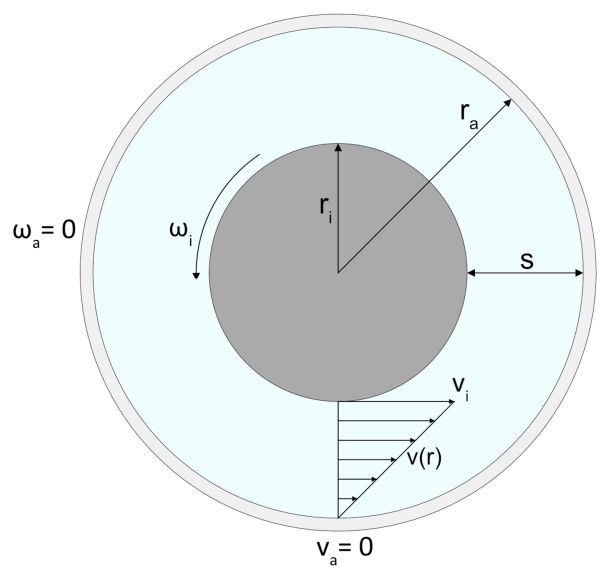

Fig. 4. Schematic representation of a cylindrical Couette flow

The liquid is moved due to the adhesion conditions and friction. Inertial forces can be neglected, and pressure and viscous forces are in balance, so the Couette flow is an accurate solution to the NavierStokes equation.

Taylor vortices occur when the velocity of the inner cylinder is increased (see Fig. 5). Due to the faster rotation, the fluid particles in the vicinity of the inner cylinder receive a greater centrifugal force than the slower particles in the outer part of the gap.

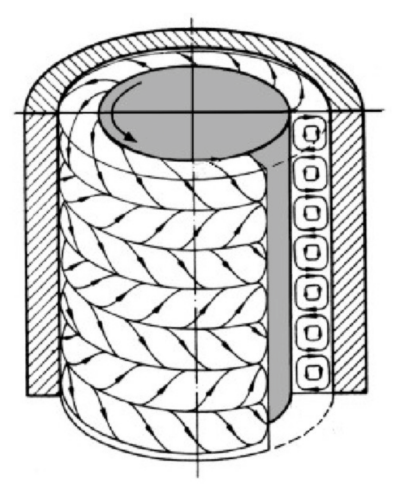

Fig. 5. Taylor-Couette flow [13] 
This corresponds to an unstable layering of a lighter medium under a heavier one (invasion). Geoffrey I. Taylor observed in 1923 that from a certain Reynolds number, regular ring vortices are superimposed on the basic flow [5]. Along the axis, these vortices occur at the same distance, but with a changing direction of rotation.

The transition from the Couette flow to the TaylorCouette flow can be calculated using the Reynolds number and is described by the Taylor number (see equation (3)). The Taylor number depends on the Reynolds number $\mathrm{Re}$ as well as on the radius of the inner cylinder $r_{i}$ and the gap width $\mathrm{s}$. For small s/d ratios, a stability analysis shows a critical Taylor number $\left(T a_{\text {critical }}=41.33\right)$ for the theoretically ideal case, so that Taylor vortices occur at higher Taylor numbers [14]. There is no clear definition in the literature for the calculation of the Taylor number. The Taylor number can be defined as follows:

$$
T a=R e \cdot \sqrt{\frac{s}{r_{i}}}[-] .
$$

The Reynolds number is calculated from the angular velocity $\omega$, the kinematic viscosity $v$ of the liquid, as well as the radius of the inner cylinder $r_{i}$ and the gap width s (see equation (4)):

$$
\operatorname{Re}=\frac{\omega \cdot\left(s \cdot r_{i}\right)}{v}[-] .
$$

The angular velocity $\omega$ is calculated according to equation (5) with the rotational speed $n$ :

$$
\omega=2 \cdot \pi \cdot n\left[\frac{1}{s}\right] .
$$

The gap width according to equation (6) is calculated using the inner and outer radius:

$$
s=r_{a}-r_{i}[\mathrm{~m}] .
$$

For the design of the test setup, the required rotational speed must first be determined for a selected geometry (see equation (7)):

$$
n=\frac{T a \cdot v}{2 \pi \cdot s \cdot r_{i} \cdot \sqrt{\frac{s}{r_{i}}}}\left[\frac{1}{s}\right] .
$$

For the Taylor number in this study, instead of the critical Taylor number $T a_{\text {critical }}=41.33$, a much higher Taylor number is used to ensure a stable Taylor Couette flow.

\subsection{Physical properties of the investigated fluid}

Due to the high complexity of a multi-phase flow, this study examines the Taylor-Couette flow with a model fluid (glycerin-water mixture), Table 1 shows the experimental results for the physical properties of the glycerin-water mixture at $20^{\circ} \mathrm{C}$. The same physical properties are used for the planned numerical flow simulation.
Tab. 1. Physical properties of the model fluid $\left(20^{\circ} \mathrm{C}\right)$

\begin{tabular}{ll}
\hline Physical properties & Value \\
\hline Density $\left[\mathrm{kg} / \mathrm{m}^{3}\right]$ & 1154 \\
Kinematic viscosity $\left[\mathrm{m}^{2} / \mathrm{s}\right]$ & $10.7 \mathrm{e}-6$ \\
Specific heat capacity $[\mathrm{j} /(\mathrm{kg} \cdot \mathrm{k})]$ & 3000 \\
Thermal conductivity $[\mathrm{w} /(\mathrm{m} \cdot \mathrm{k})]$ & 0.383 \\
Thermal diffusivity $\left[\mathrm{m}^{2} / \mathrm{s}\right]$ & $1.1 \mathrm{e}-7$ \\
\hline
\end{tabular}

Important for measurements with Ultrasonic Doppler Velocimetry is the sound velocity at the time of measurement. The UDV measurement technique can be modified and used for this purpose based on a transit time difference method for the exact measurement of the sound velocity.

\section{DIMENSIONLESS NUMBERS OF THE FLOW}

The most important dimensionless numbers used in this study for the selected boundary conditions of the experiment are listed in Tab. 2 below.

Tab. 2. Example of Dimensionless numbers

\begin{tabular}{lll}
\hline Numbers & Relevant parameters & Value \\
\hline Prandtl & Glycerin-water mixture at $20^{\circ} \mathrm{C}$ & 96.4 \\
Reynolds & $100 \mathrm{rpm}$ & 514 \\
Taylor & $R e, s=15 \mathrm{~mm}, r_{i}=35 \mathrm{~mm}$ & 336 \\
\hline
\end{tabular}

\section{RESULTS AND DISCUSSION}

\subsection{Measurement of velocity profile with UDV}

The velocity profile was measured continuously every 5 minutes. The sound velocity required for the UDV measurement system was determined periodically by using a transit time method in the Taylor-Couette system and averaged at approx. 1819 $\mathrm{m} / \mathrm{s}$. In the first measurements, the vertical measuring axis of the UDV is aligned approx. $3 \mathrm{~mm}$ beside the axis by the Taylor vortex centres in order to obtain an alternating velocity profile [15-19].

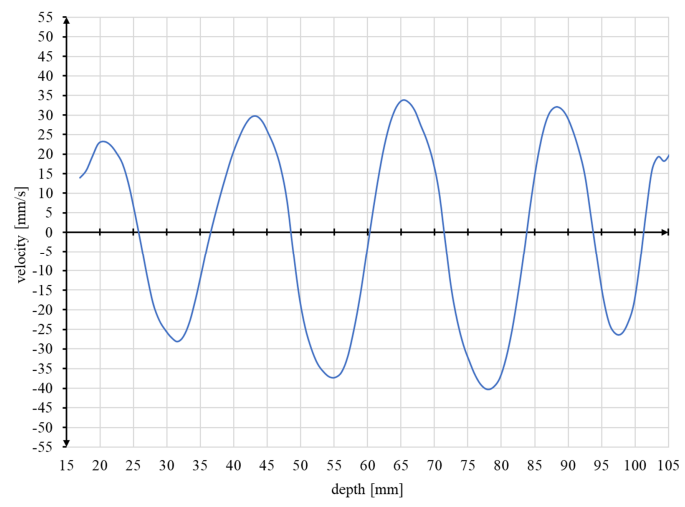

Fig. 6. Example of a velocity profile of the Ultrasonic Doppler measuring technique in $60 \%$ glycerine-water mixture at $100 \mathrm{rpm}$ 


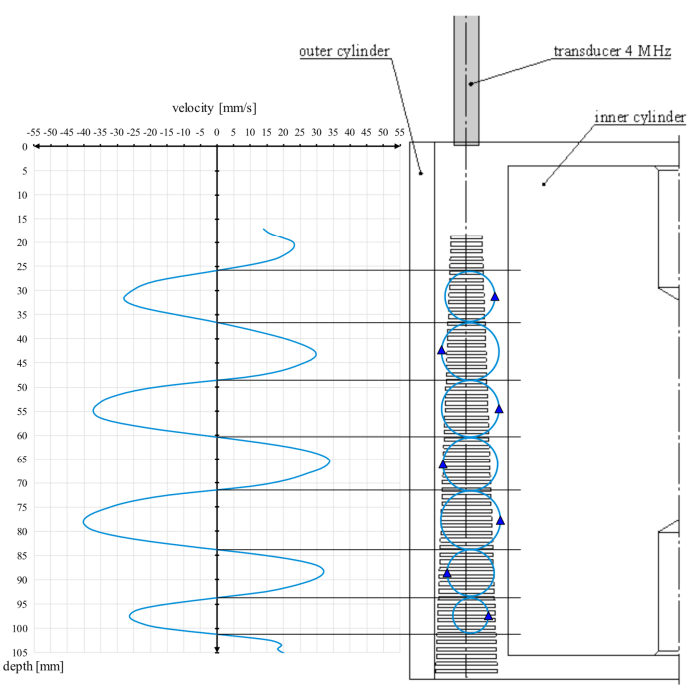

Fig. 7. Schematic representation of the resulting Taylor vortices

The profile shown in Fig. 6 could be determined in each of the 100 measurements with small deviations, especially in the ranges of local maxima or minima. The resulting flow pattern can be considered stable under stationary operating conditions, as the measurements were performed over a long period of time (3 hours). Furthermore, a minimal increase in the velocities (max. approx. $40 \mathrm{~mm} / \mathrm{s}$ ) and size of the Taylor vortices towards the centre can be observed. This change is due to the relatively short design of the system and the influence of the top and bottom plates. Fig. 7 gives an idea of how many Taylor vortices form in the gap.

\subsection{Measurement of velocity profile with LDA}

For velocity measurements by Laser Doppler anemometry, an area in the gap was required in which measurements could be carried out accurately and without interference. Such disturbances can be, for example, reflections on the shafts screwed into the inner cylinder or on the inner cylinder itself. By means of traversing the measuring lines can be traversed in vertical and horizontal direction [20].

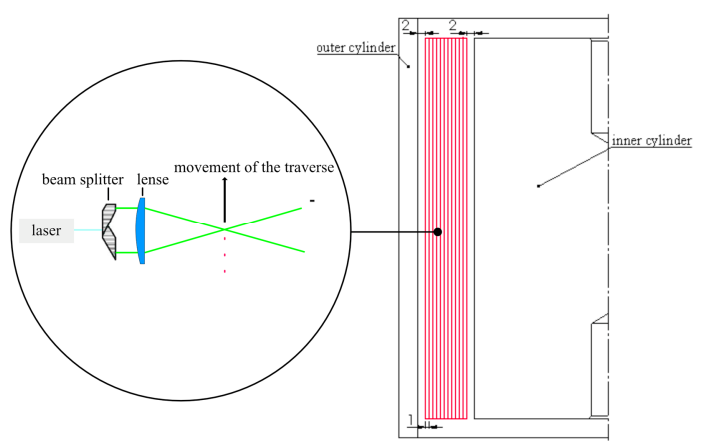

Fig. 8. Schematic representation of the measuring field with the LDA
In order for the signal strength to be sufficiently high, it is important to pay attention to a strong seeding. Due to the very small measuring volume (approx. $140 \mu \mathrm{m}$ ) and the high sampling rates, a spatial and temporally highly accurate resolution is possible. Fig. 9 shows an example of the measured velocity profile at a distance of 4 millimetres from the inner surface of the outer cylinder at a rotational speed of $150 \mathrm{rpm}$. With increasing rotational speed, the fluctuations also increase in the velocities of the Taylor vortices. This can be seen particularly well in the strongly fluctuating axial speeds of the vortices. A possible reason here could be the transition to the wavy Taylor Couette flow, which occurs at higher Reynolds and leads to an additional axial movement of the torus-shaped vortex rolls.

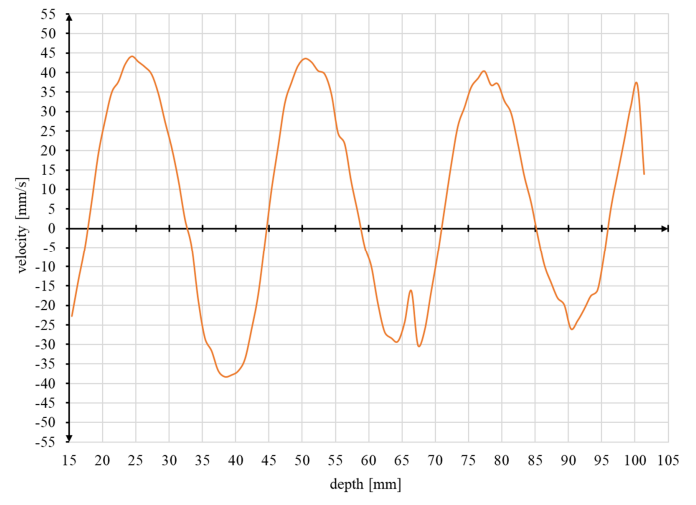

Fig. 9. Example of a measured LDA profile in $60 \%$ glycerin-water mixture at a distance of 4 millimetres from the inner surface of the outer cylinder (150 rpm)

\subsection{Comparison of the LDA and UDV measurements}

The laser Doppler anemometry measurements were performed simultaneously with the Ultrasonic Doppler measurements. The LDA served as a reference to study the signal behaviour of the UDV due to its very reliable and accurate measuring principle. The constant rotational speed of the inner cylinder is $150 \mathrm{rpm}$. The measurements were only started after a very long period of stationary operation. The settings for the Ultrasonic Doppler measurement technique were 1000 values at a frequency of 4 megahertz per measurement volume. Fig. 10 shows the average value of 5 selected measurements with the Ultrasonic Doppler measurement technique as well as the result of the measurement with the laser Doppler anemometry. The measuring axis of the LDA is located at a distance of 4 millimetres from the inner surface of the outer cylinder. 


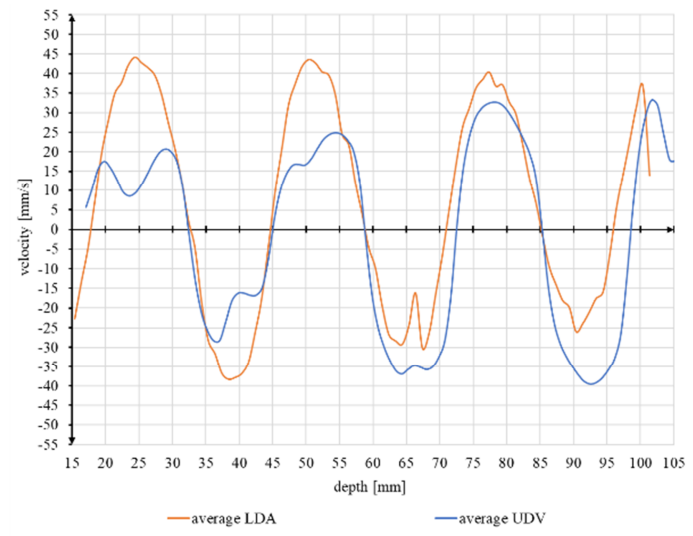

Fig. 10. Average value of 5 UDV measurements and measured velocity profile using the LDA

If one considers the distribution of velocity in a single vortex cell, starting from the vortex centre, it increases with increasing distance from the vortex centre in the direction of the cylinder walls.

Both measurement techniques reproduce this well in terms of quality. The comparison clearly shows the exact position of the vortex centres and the axial position of the minima and maxima in both techniques. The quantitative agreement shows deviations, which are caused e.g. by the different size of the measuring volume.

Since the measuring volume of the Ultrasonic Doppler measuring technique is significantly larger compared to laser Doppler anemometry, velocities of different magnitudes are detected and used for averaging.

In this way, it is possible to obtain lower average velocities. The measurement of laser Doppler anemometry, on the other hand, have more character of a point measurement. The LDA profile shown is focused closer to the outer cylinder than the UDV profile. Therefore, higher vortex velocities are partly measured.

\section{CONCLUSION AND OUTLOOK}

In the present study a test setup was built to generate a stable reproducible flow based on a Taylor Couette flow. In this flow configuration, velocity profile measurements and the signal behaviour of the ultrasonic Doppler measurement technique could be comprehensively evaluated. The measurements were performed in a transparent model fluid and compared in parallel with an optical measurement technique (LDA), which allows precise high-resolution measurements.

In general, there was a good qualitative agreement between the measurements and structural parameters of the Taylor vortices, which could be recorded very well. However, there were also deviations in the size of the measured values. This is probably caused by the arrangement of the measuring axes and the different sizes of the measuring volumes of the two measuring techniques.

In further experimental investigations as well as numerical simulations it is planned to investigate this signal behaviour comprehensively, in order to be able to interpret the results of the UDV measurements more effectively.

\section{Acknowledgements}

The authors gratefully acknowledge by the "European Regional Development Fund" (ERDF) for the financial support for the measurement technique "Ultrasonic Velocimetry System" (GHS-170021).

\section{Nomenclature}

\section{Symbols}

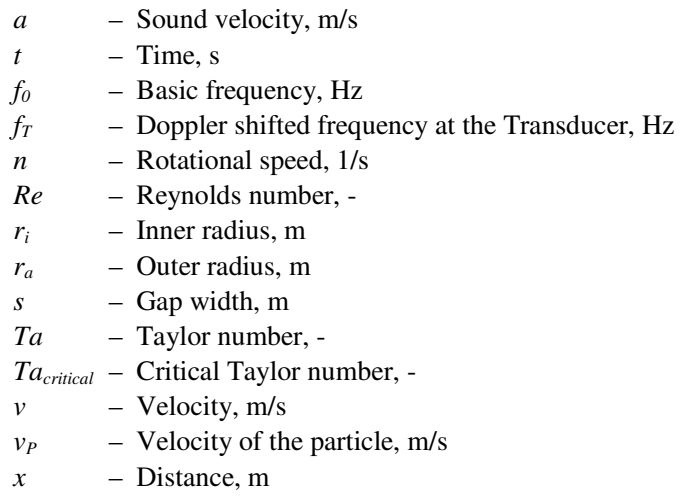

\section{Greek letters}

$v \quad-$ Kinematic viscosity, $\mathrm{m}^{2} / \mathrm{s}$

$\omega \quad-$ Angular velocity, $1 / \mathrm{s}$

\section{Acronyms}

LDA - Laser Doppler Anemometry

PIV - Particle Image Velocimetry

rpm - Revolutions per minute

UDV - Ultrasonic Doppler Velocimetry

\section{References}

1. Klembt D., Meironke H. (2018) Experimental investigations of the influence of different bottom shapes on the temperature and velocity fields in a fermentation tank with a biological multiphase flow, In Proceedings 11. International Symposium on Ultrasonic Doppler Methods for Fluid Mechanics and Fluid Engineering, Berlin.

2. Meironke H., Klembt D., Panten T. (2017). Untersuchungen zum Einfluss von Gasblasen auf die Ultraschall-Doppler-Messtechnik mittels optischer und numerischer Methoden, In: B. Ruck, C. Gromke, A. Leder, D. Dopheide (ed.), Lasermethoden in der Strömungsmesstechnik, 25. Fachtagung der GALA e.V., Karlsruhe, ISBN 978-3-9816764-3-3, pp. 45.1 -45.8

3. Klembt D., Meironke H., Pommer E.: Untersuchungen zum Einfluss von Blasensäulen auf die Ultraschall Doppler Messtechnik, In: S. Grundmann, M. Brede, B. Ruck, A. Leder, D. Dopheide (ed.), Lasermethoden in der Strömungsmesstechnik, 26. Fachtagung der GALA e.V., Rostock, ISBN 978-3-9816764-5-7, pp. 7.1 -7.8 
4. Klembt D., Meironke H., Delgado A. (2019). Untersuchungen zum Einfluss von Blasenschwärmen und -säulen auf die Ultraschall Doppler Messtechnik, in: A. Delgado, B. Ruck, D. Dopheide (ed.), Lasermethoden in der Strömungsmesstechnik, 27. Fachtagung der GALA e.V., Erlangen.

5. Taylor G. I. (1923). Stability of a Viscous Liquid Contained between Two Rotating Cylinders, Vol. 223. London: The Royal Society of London, pp. 289-343.

6. Andereck C. D., Lius S. S., Swinney H. L. (1986). Flow regimes in a circular Couette system with independently rotating cylinders. Journal of Fluid Mechanics, Vol. 164, pp. $155-183$

7. Mizushima J., Suehiro N. (2005). Instability and transition of flow past two tandem circular cylinders, Physics of Fluids, Vol. 17, doi:doi: 10.1063/1.2104689.

8. Donnelly R. J. (1991). Taylor-Couette Flow: The early days, In Physics Today, American Institute of Physics, pp. 32-39.

9. Ng B.S., Turner E.R., (1982). On the linear stability of spiral flow between rotating cylinders, Proceedings Royal Society London, Ser. A 382, pp. 83-102.

10. Moore C.M.V. (1994). Characterization of a TaylorCouette vortex flow reactor, $P h D$ Thesis, Dept. of Chem. Eng., MIT.

11. Davey A. J. (1962). The growth of Taylor vortices in flow between rotating cylinders, Fluid Mechanics, Vol. 14, pp. 336-368.

12. Escudier M. P., Gouldson I. W., Jones D. M. (1994). Circular Couette Flow and Taylor Vortices in ShearThinning Liquids, In Adrian R. J. et al. (ed.) Development in Laser Techniques and Applications to Fluid Mechanics, Springer Verlag, Berlin.

13. Claßens D., Faouzi H., Mayer D. (2009). Numerische Simulation einer Taylor-Couette Strömung und Modellierung thrombozytärer Reaktionen. Aachen: RWTH Aachen CATS.

14. Oertel jr., Delfs J. (1996). Strömungsmechanische Instabilitäten, Springer Verlag, ISBN: 3-540-56984-7.

15. Takeda Y., Ficher W. E, Sakakibara J., Ohmura K. (1993). Experimental observation of the quasiperiodic modes in a rotating Couette system, Phys Rev E47(6), pp. 4130-4134.

16. Takeda Y. (1999). Quasi-periodic state and transition to turbulence in a rotating Couette system, Journal of Fluid Mechanics, Vol. 389, pp 91-99.

17. Racina A. (2008). Vermischung in Taylor-Couette Strömung, PhD University Karlsruhe.

18. Kikura H., Takeda Y., Durst F. (1991). Velocity profile measurement of the Taylor vortex flow of a magnetic fluid using the ultrasonic Doppler method, Experiments in Fluids, Vol. 26, ISSN 1432-1114, pp. 208-214.

19. Takada D., Takeda Y. (2003). Oscillating Taylor-Couette Flow, Hokkaido University.

20. Parker J., Merati P. (1996). An investigation of turbulent Taylor-Couette flow using Laser-Doppler velocimetry in a refractive index matched facility, Journal of Fluids Engineering, Vol. 118, pp. 810-818.

\section{Biographical notes}

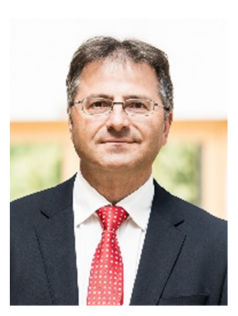

Heiko Meironke received his doctoral degree (Dr.-Ing.) at the University of Rostock in the Faculty of Mechanical Engineering and Shipbuilding Technology, specialising in applied mechanics, and is currently professor at the University of Applied Sciences Stralsund. Since 2009 he is leader of the Department of Fluid Mechanics and Apparatus Engineering at the University of Applied Sciences Stralsund. His scientific interests are the investigation of general fluid mechanics problems, in particular the experimental and numerical investigation of multiphase flows in fermenting fluids. He presented these topics regularly at several national and international conferences and published the results in several conference proceedings and journals.

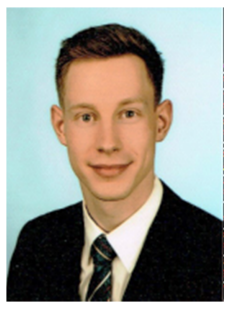

Daniel Klembt is a mechanical engineer and is currently completing his doctoral degree (Dr.-Ing.) on real biological multiphase flows at the University Erlangen-Nürnberg. Since 2017 he has been a research assistant at the Department of Fluid Mechanics and Apparatus Engineering at the University of Applied Sciences Stralsund. His scientific interests are in the fields of acoustic velocity profile measurement, optical measurement techniques, numerical simulation and generally the investigation of real multiphase flows. He participated in 5 international and 3 national conferences and presented the results of his work, published more than 10 scientific papers in international and national journals, book chapters and conference reports. 
